\title{
Bessel beam Grueneisen photoacoustic microscopy with extended depth of field
}

Junhui Shi, Lidai Wang, Cedric Noordam, Lihong V. Wang

Junhui Shi, Lidai Wang, Cedric Noordam, Lihong V. Wang, "Bessel beam Grueneisen photoacoustic microscopy with extended depth of field," Proc. SPIE 9708, Photons Plus Ultrasound: Imaging and Sensing 2016, 97083H (15 March 2016); doi: 10.1117/12.2211198

SPIE. Event: SPIE BiOS, 2016, San Francisco, California, United States 


\title{
Bessel Beam Grueneisen photoacoustic Microscopy with Extended Depth of Field
}

\author{
Junhui Shi ${ }^{\mathrm{a}^{\dagger}}$, Lidai Wang ${ }^{\mathrm{a}, \mathrm{b} \dagger}$, Cedric Noordam ${ }^{\mathrm{a}, \mathrm{c}}$, and Lihong V. Wang ${ }^{\mathrm{a}^{*}}$ \\ ${ }^{a}$ Optical Imaging Laboratory, Department of Biomedical Engineering, Washington University in \\ St. Louis, St. Louis, Missouri 63130-4899, USA \\ ${ }^{\mathrm{b}}$ Current affiliation: Department of Mechanical and Biomedical Engineering, \\ City University of Hong Kong, 83 Tat Chee Ave, Kowloon, Hong Kong SAR, China \\ ${ }^{\mathrm{c}}$ Current affiliation: Optical Sciences, MESA+ Institute for Nanotechnology, \\ University of Twente, P. O. Box 217, 7500 AE Enschede, The Netherlands \\ *Corresponding author, LHWANG@,WUSTL.EDU. ${ }^{\dagger}$ Equal contributions.
}

\begin{abstract}
:
The short focal depth of a Gaussian beam limits the volumetric imaging speed of optical resolution photoacoustic microscopy (OR-PAM). A Bessel beam, which is diffraction-free, provides a long focal depth, but its side-lobes may deteriorate image quality when the Bessel beam is directly employed to excite photoacoustic signals in ORPAM. Here, we present a nonlinear approach based on the Grueneisen relaxation effect to suppress the side-lobe artifacts in photoacoustic imaging. This method extends the focal depth of OR-PAM and speeds up volumetric imaging. We experimentally demonstrated a $1-\mathrm{mm}$ focal depth with a $7-\mu \mathrm{m}$ lateral resolution and volumetrically imaged a carbon fiber and red blood cell samples.
\end{abstract}

Keywords: Photoacoustic, Bessel beam, Grueneisen relaxation, nonlinear microscopy

\section{INTRODUCTION}

Optical resolution photoacoustic microscopy (OR-PAM), which provides high sensitivity by detecting optical absorption contrasts, has been widely used for imaging biological systems, from single cells to organisms [1-5]. A conventional OR-PAM setup typically excites molecules using a focused Gaussian laser beam, which can have a micron- to sub-micron-sized focal spot but suffers from limited focal depth. In order to acquire mm-thick volumetric images with consistent lateral resolution in the depth direction, we need three-dimension raster scanning to compensate for the short focal depth, which reduces the imaging speed. In comparison, a Bessel beam is essentially diffraction-free, i.e. the focal depth can be extended greatly without compromising the focal spot size, which avoids the tradeoff between imaging resolution and focal depth [6-8]. However, Bessel beams have many strong side lobes, which generate ghost signals, limiting their application in optical imaging. Similarly in photoacoustic imaging, ultrasound transducers usually have large focal diameters (tens to hundreds of microns), and thus they receive most of the ghost signals from the side lobes of the Bessel beam [9], which inhibits the direct application of Bessel beams in photoacoustic imaging.

Until recently, nonlinear optical techniques, e.g. two-photon excitation in fluorescence microscopy, have been successfully used to suppress the side-lobe effect of Bessel beams and then achieve rapid volumetric imaging with a long focal depth [10-14]. Nonlinear effects exist in photoacoustic imaging as well [15-21]. One of them is the Grueneisen relaxation effect [20]. a thermally induced nonlinear effect that has been explored to improve both the axial and lateral resolution for PAM [20,21]. Here, we utilize the Grueneisen relaxation effect in Bessel-beam PAM to suppress the side-lobe effect and achieve long-focal-depth volumetric imaging.

\section{METHODS}

Grueneisen relaxation photoacoustic microscopy (GR-PAM) employs dual-pulse excitations to obtain nonlinear photoacoustic signals [20]. The first laser pulse generates a photoacoustic signal and, at the same time, increases the local temperature. The second laser pulse is almost identical to the first laser pulse and excites the same absorber within the thermal confinement time. Owing to the temperature rise induced by the first pulse, the local Grueneisen parameter is increased, and the second photoacoustic signal is stronger than the first one. The nonlinear photoacoustic signal is the amplitude difference between these two signals, and is proportional to the square of the

Photons Plus Ultrasound: Imaging and Sensing 2016, edited by Alexander A. Oraevsky, Lihong V. Wang Proc. of SPIE Vol. 9708, 97083H - (c) 2016 SPIE · CCC code: 1605-7422/16/\$18 · doi: 10.1117/12.2211198 
optical fluence, thereby achieving nonlinear effects. Alternatively, we can also realize GR-PAM using a two-step measurement scheme. In the first step, one probe laser pulse generates a photoacoustic signal; in the second step, a heating laser is applied before firing the second probe laser pulse. The resulting nonlinear photoacoustic signal is the difference of the two signals from the two probe laser pulse excitations. In this way, the heating laser could be either a continuous-wave (CW) or a pulsed laser, as long as the turn-on time and time delay between the heating and probing fulfill the thermal confinement requirement. In the two-step measurement scheme, the first probe laser pulse generates an initial pressure rise,

$$
p_{1}(x, y)=\Gamma_{0} \eta_{t h} \mu_{a}(x, y) F_{1}(x, y),
$$

where $\Gamma_{0}$ is the Grueneisen parameter at the initial temperature, $\eta_{t h}$ is the heat conversion efficiency, $\mu_{\mathrm{a}}(x, y)$ is the optical absorption coefficient, and $F_{1}(x, y)$ is the optical fluence of the probe pulse laser. The photoacoustic signal is the spatial integration of $p_{1}(x, y)$,

$$
\mathrm{PA}_{1}=k \Gamma_{0} \eta_{t h} \iint \mu_{a}(x, y) F_{1}(x, y) d x d y,
$$

where $k$ is a coefficient representing the ultrasonic detection sensitivity and is assumed to be spatially invariant within the acoustic focus.

In the second step, after the heating laser is applied, the Grueneisen parameter increases owing to the local temperature rise,

$$
\Gamma_{2}=\left[1+\alpha \eta_{t h} \mu_{a}(x, y) F_{2}(x, y)\right] \Gamma_{0},
$$

where $\alpha$ is a constant that relates the absorbed energy to the Grueneisen parameter change, and $F_{2}(x, y)$ is the optical fluence of the heating laser. The initial pressure rise induced by the second probe laser pulse is given by

$$
p_{2}(x, y)=\left[1+\alpha \eta_{t h} \mu_{a}(x, y) F_{2}(x, y)\right] \Gamma_{0} \eta_{t h} \mu_{a}(x, y) F_{1}(x, y) .
$$

The corresponding photoacoustic signal is

$$
\mathrm{PA}_{2}=k \Gamma_{0} \eta_{t h} \iint \mu_{a}(x, y)\left[1+\alpha \eta_{t h} \mu_{a}(x, y) F_{2}(x, y)\right] F_{1}(x, y) d x d y .
$$

Subtracting Eq. (2) from Eq. (5), we obtain a differential photoacoustic signal, $\Delta$ PA,

$$
\Delta \mathrm{PA}=k \Gamma_{0} \alpha \eta_{t h}^{2} \iint \mu_{a}^{2}(x, y) F_{2}(x, y) F_{1}(x, y) d x d y .
$$

When the heating laser and probe laser have the same optical beam profile, then their optical fluences are linearly proportional to each other: $F_{2}(x, y)=g F_{1}(x, y)$, where $g$ is a constant depending on the power ratio of these two lasers. This simplifies Eq. (6) to

$$
\Delta \mathrm{PA}=g k \Gamma_{0} \alpha \eta_{t h}^{2} \iint \mu_{a}^{2}(x, y) F_{1}^{2}(x, y) d x d y,
$$

which shows a nonlinear dependence on optical fluence, while the signal $\mathrm{PA}_{1}$ has only a linear dependence as expressed in Eq. (2).

Let the optical fluence follow a Bessel profile,

$$
I(r, z)=I_{0}(z) J_{0}^{2}\left(2.4048 \frac{r}{r_{0}}\right)
$$

where $I_{0}(z)$ is the optical intensity at axial position $z, r_{0}$ is the radius of the central lobe, $r$ is the transverse radial coordinate $\left(r=\sqrt{x^{2}+y^{2}}\right)$ and $J_{0}(r)$ is the zero-order Bessel function of the first kind. Due to the existence of many side-lobes, the point spread function of linear OR-PAM on the transverse plane following the function of 
$J_{0}^{2}(r)$ also has many side-lobes. But if we use the nonlinear photoacoustic signal $\Delta \mathrm{PA}$ to form an image, then the point spread function becomes the square of the Bessel beam profile, $J_{0}^{4}(r)$. Because the amplitudes of side lobes are much smaller than that of the central lobe, the contribution of the side lobes is reduced by squaring, as has been demonstrated in two-photon Bessel-beam microscopy [10]. The attenuation of side-lobe effects in nonlinear Besselbeam PAM can also be interpreted as a result of low heating effect of the side-lobes due to their low optical fluence. The further away the side lobe is from the central lobe, the less the nonlinear photoacoustic signal it contributes.

\section{EXPERIMENTAL SETUP}

Fig. 1A is a schematic of a Bessel-beam PAM system. The probe laser is a $532 \mathrm{~nm}$ diode-pumped solid-state pulsed laser (Innoslab BX2II-E, Edgewave $\mathrm{GmbH}$ ), which generates $10 \mathrm{~ns}$ laser pulses at repetition rates in the range of hundreds of $\mathrm{Hz}$. The heating laser beam, from either a $\mathrm{CW}$ laser or a pulsed laser, is combined with the probe laser beam and then spatially filtered by a 50- $\mu \mathrm{m}$ tungsten pinhole. The filtered laser beam has a 3-mm diameter and a near-Gaussian transverse profile. Finally, an axicon lens (Thorlabs AX255-A, $25 \mathrm{~mm}, 5.0^{\circ}$ ) transforms the laser beam into a Bessel beam. The sample holder, a petri dish filled with water for ultrasound coupling, is mounted on a 3D scanning stage (PLS-85, PI miCos $\mathrm{GmbH}$ ) and it is about $7 \mathrm{~mm}$ away from the tip of the axicon lens. When the sample is irradiated by the Bessel beam, photoacoustic waves are generated through light absorption, and then detected by a custom-made focused ultrasonic transducer ( $48 \mathrm{MHz}$ central frequency, $80 \%$ detection bandwidth, $11.6 \mathrm{~mm}$ focal length, and $0.25 \mathrm{NA}$ ). The focal zone of this transducer is about $2 \mathrm{~mm}$, encompassing the long focal depth of the Bessel beam. The photoacoustic signal from the transducer is further amplified by $40 \mathrm{~dB}$ (ZKL-1R5+, Minicircuits) and digitized at $500 \mathrm{MHz}$ using a data acquisition card (ATS9350, AlazarTech). A LabVIEW programed FPGA card (PCI-7830R, National Instruments) synchronizes the laser triggers, scanner motion, and data acquisition for imaging.

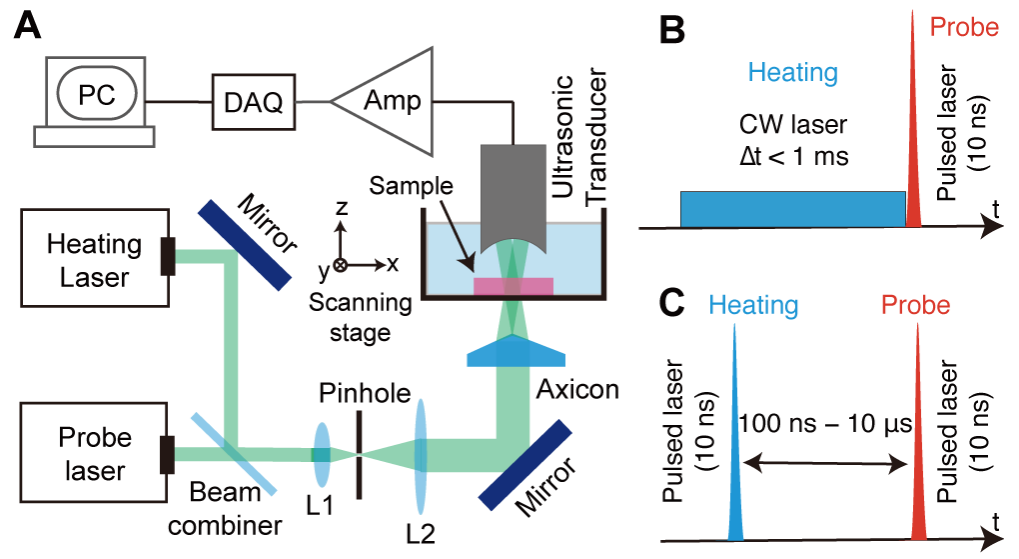

Fig. 1. Schematic of Bessel-beam PAM. (A) Experimental system setup. L1, L2: lens. (B) CW-heating scheme. A continuous wave $(\mathrm{CW})$ laser is used as the heating laser. The heating duration $(\Delta \mathrm{t})$ of the $\mathrm{CW}$ laser must be less than the thermal relaxation time. (C) Pulsed-heating scheme. Both the heating and probe laser beams are pulsed, and the interpulse delay ranges from $100 \mathrm{~ns}$ to $10 \mu \mathrm{s}$.

The axicon lens converts a Gaussian beam to a good approximation of a true Bessel beam [10,22-24]. According to Ref. [25], the radius of the central lobe $(\rho)$ is defined as the first zero of the Bessel function: $\rho=2.40 \lambda /(2 \pi \beta)$, where $\lambda$ is the wavelength, $\beta=\sin ^{-1}(n \sin \alpha)-\alpha, \alpha$ is the axicon angle and $n$ is the refractive index of the axicon lens material. The depth of field is $L=0.8 w_{0} / \beta$, where $w_{0}$ is the beam diameter of the incident Gaussian beam. In our system, the wavelengths of both the $\mathrm{CW}$ laser and pulsed lasers are $532 \mathrm{~nm}$, and the beam diameter of the incident Gaussian beam is $3 \mathrm{~mm}$. The beam profile, measured by a beam profiler (BeamGage, Ophir) as shown in Fig. 2A, is the transverse intensity profile of the Bessel beam taken at a relative axial position $\mathrm{z}=2 \mathrm{~mm}$ (The axial position at $\mathrm{z}=0 \mathrm{~mm}$ is about $7 \mathrm{~mm}$ away from the tip of the axicon lens). The central lobe has a Full Width at Half Maximum (FWHM) of $6.5 \mu \mathrm{m}$, while the side lobes spread widely beyond a 100- $\mu \mathrm{m}$-diameter circular area. The central lobe diameter varies depending on the axial position relative to the axicon lens. The axial distributions of the central lobe diameter (FWHM) and peak intensity of the central lobes are shown in Fig. 2B. In the region $\mathrm{z}>0 \mathrm{~mm}$, the transverse beam profile can be described as a quasi-Bessel beam, and the central lobe diameter is around 6-7 $\mu \mathrm{m}$. In the region $\mathrm{z}<0$, i.e., closer to the axicon lens, the central lobe diameter diverges quickly; hence this region 
should be avoided for imaging, due to its poor resolution. In practice, the peak intensity is also an important factor, since a low peak intensity not only attenuates the photoacoustic signal, but also attenuates the heating effect. Therefore, for the Bessel beam shown in Fig. 2, only a 2-mm focal depth $(0<\mathrm{z}<2 \mathrm{~mm})$ is used for nonlinear Bessel-beam PAM. This focal depth is much larger than that of conventional Gaussian beams, e.g., the focal depth of a Gaussian beam with the same focal spot size $6 \mu \mathrm{m}$ at $532 \mathrm{~nm}$ is only about $106 \mu \mathrm{m}$.

A

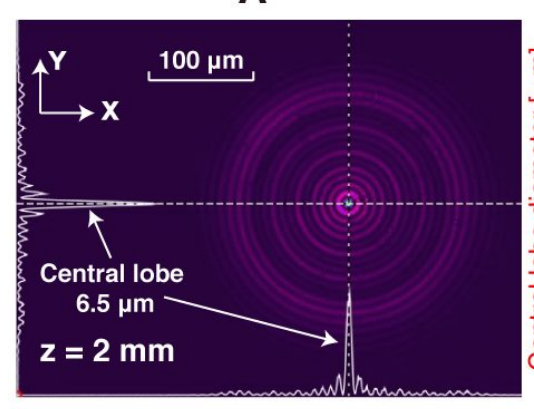

B

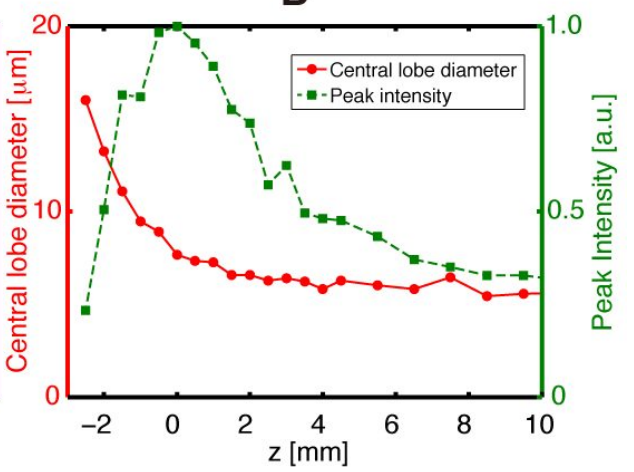

Fig. 2. Bessel beam profile. (A) Transverse intensity profile taken at $\mathrm{z}=2 \mathrm{~mm}$ (at $\mathrm{z}=0 \mathrm{~mm}$, it is about $7 \mathrm{~mm}$ away from the tip of the axicon lens). The central lobe diameter $(6.5 \mu \mathrm{m})$ is the FWHM of the central lobe. White peaks represent intensity profiles along white dashed lines respectively. (B) Axial distributions of the central lobe diameter (red solid line, Y-axis is on the left side) and peak intensity (green dashed line, Y-axis is on the right side).

To generate non-linear PA signals, we employ two schemes. In the first scheme, as illustrated in Fig. 1B, a CW laser is turned on for a short period of time $\Delta \mathrm{t}$ to heat the sample, then a pulsed probe laser is triggered at the end of the heating. The heating time is within the thermal relaxation time. The pulsed probe laser generates PA signals, while the $\mathrm{CW}$ laser is only for heating. At each scanning point, we record two PA signals: The first PA signal $\left(\mathrm{PA}_{1}\right)$ is excited by a single laser pulse without $\mathrm{CW}$ laser heating, while the second signal $\left(\mathrm{PA}_{2}\right)$ is excited by another laser pulse right after the $\mathrm{CW}$ laser heating. Owing to the Grueneisen relaxation effect, the second PA signal is stronger than the first one, and their amplitude difference is the non-linear PA signal $(\Delta \mathrm{PA})$. Due to its low power $(<100 \mathrm{~mW}$ in our system) and the thermal confinement of the target, the $\mathrm{CW}$ laser is good for heating highly absorptive samples (e.g. carbon fiber, hair). In the second scheme, both the heating laser and the probe laser are pulsed, as shown in Fig. 1C. The inter-pulse delay is a constant ranging from $100 \mathrm{~ns}$ to several $\mu \mathrm{s}$, which is much shorter than the thermal relaxation time. In this scheme, similar to the first scheme, two probe PA signals are recorded with and without the pulsed heating. Their computed amplitude difference is the non-linear PA signal $(\triangle \mathrm{PA})$. The pulsed heating laser, possessing higher pulse energy, provides better heating effect for low absorptive samples but is more expensive than the $\mathrm{CW}$ heating laser. In our experiments, we demonstrated the feasibility of both schemes. We employed the first scheme to measure a carbon fiber to quantify the effect of the nonlinear Bessel-beam PAM, and then used the second scheme to image red blood cell samples.

\section{RESULTS}

In order to quantify the lateral resolution and effective axial focal depth, we imaged a carbon fiber (6- $\mu$ m diameter) using the nonlinear Bessel-beam PAM system. The thermal relaxation time of a carbon fiber immersed in water with a $6.5-\mu \mathrm{m}$ diameter optical heating spot has been estimated to be $1 \mathrm{~ms}$ [26], so we heated the carbon fiber using a 532 $\mathrm{nm} \mathrm{CW}$ laser ( $40 \mathrm{~mW}$ power, $300 \mu$ s duration, equivalent heating energy $12 \mu \mathrm{J}$ ), and excited PA signals using a pulsed laser (10 ns pulse width, $1200 \mathrm{~nJ}$ pulse energy). Because the energy distributes almost equally among the central and each side lobe of the Bessel beam, only a small portion of the energy can be absorbed by the thin carbon fiber. The carbon fiber was placed horizontally in the petri dish and immersed in water for coupling, and a crosssectional photoacoustic image was obtained by one-dimensional mechanical scanning across the carbon fiber. A typical measured photoacoustic amplitude profile across the carbon fiber is shown in Fig. 3A, where the red line $\mathrm{CW}_{\text {on }}$ is the enhanced PA image with the $\mathrm{CW}$ laser heating, while the blue line $\mathrm{CW}_{\text {off }}$ is the conventional PA image acquired without laser heating. Both the $\mathrm{CW}_{\text {on }}$ and $\mathrm{CW}_{\text {off }}$ images have significant backgrounds, which are produced by the side lobes of the Bessel beam. By taking the amplitude difference between $\mathrm{CW}_{\text {on }}$ and $\mathrm{CW}_{\text {off }}$, we computed the differential photoacoustic image $\triangle \mathrm{PA}$, which clearly reduces the background and suppresses the side lobe effect. In 
Fig. $3 \mathrm{~A}$, the FWHM of $\triangle \mathrm{PA}$ is about $6.1 \mu \mathrm{m}$, which is close to the value of $6.5 \mu \mathrm{m}$ estimated from the convolution of the carbon fiber cross-section profile and the profile squared of the Bessel beam central lobe. Fig. 3B shows the lateral resolution at different axial positions. In the region of $0.6 \mathrm{~mm}<\mathrm{z}<2.2 \mathrm{~mm}$, the FWHM value is around 6.2 $\mu \mathrm{m}$, while it increases quickly for smaller $\mathrm{z}(<0.6 \mathrm{~mm}$, closer to the axicon lens) because the central lobe diameter becomes large, as shown in the Bessel light beam profile in Fig. 2B. For $\mathrm{z}>2.2 \mathrm{~mm}$ (further from the axicon lens), the FWHM also deteriorates, since the heating light intensity of the central lobe is attenuated significantly in this region. In summary, the effective nonlinear Bessel-beam imaging region in Fig. 3B is about $1.6 \mathrm{~mm}$, in which the system maintains a lateral resolution finer than $\sim 7 \mu \mathrm{m}$.
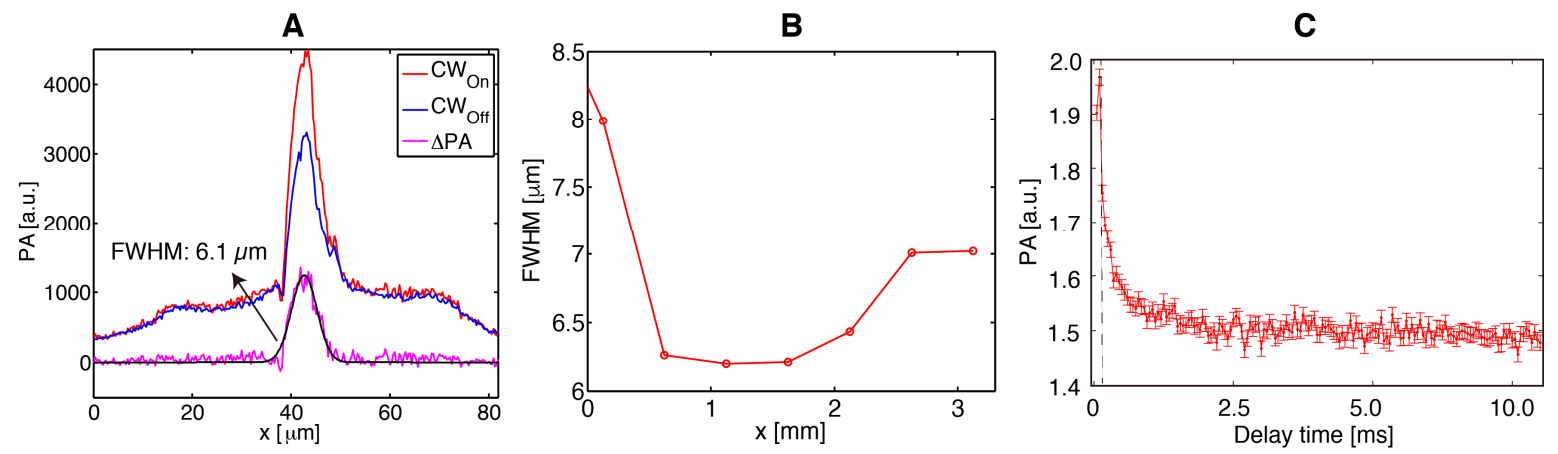

Fig. 3. Cross-sectional images of a 6- $\mu$ m-diameter carbon fiber. (A) Red line: 1D photoacoustic image acquired when the CW laser is on. Blue line: 1D photoacoustic image acquired when the CW laser is off. Green line: the differential photoacoustic image $\triangle \mathrm{PA}$. Black line: Gaussian fit of $\triangle \mathrm{PA}$. The FWHM of the $\triangle \mathrm{PA}$ image is $6.1 \mu \mathrm{m}$. To improve SNR, 20 measurements were averaged. (B) Axial distribution of the FWHM of the cross-sectional carbon fiber images. The axial coordinate $\mathrm{z}$ is relative to the beginning measurement position ( $\mathrm{z}=0$ is about $7 \mathrm{~mm}$ away from the tip of the axicon lens). (C) PA amplitude versus time delay of the laser pulse after a $0.1 \mathrm{~ms} \mathrm{CW}$ laser irradiation.

Besides the carbon fiber, we also imaged red blood cell (RBC) samples, which are relatively low absorptive. Bovine RBCs (Quad Five, Ryegate, Montana) were first fixed with methanol and then mixed with agar gel (1\% weight ratio of agarose and water) at $40{ }^{\circ} \mathrm{C}$. The volume ratio between the $\mathrm{RBC}$ and agar gel was 1:500. A single-layer RBC sample was prepared by simply smearing the RBC-agar gel onto a cover glass. A thick-layer $(0.5 \mathrm{~mm}$ to $1.0 \mathrm{~mm})$ RBC sample was formed by dropping the gel into a hole in the bottom of a petri dish sealed with a cover glass. Finally, the RBC-agar gel was cooled to room temperature $\left(25^{\circ} \mathrm{C}\right)$ to form solid samples. The single-layer RBC sample was used to quantify the side-lobe suppression effect. The thick-layer sample was used to demonstrate the volumetric imaging capability of nonlinear Bessel-beam PAM. To provide sufficient power to heat the highly diluted RBC sample, we exchanged the $\mathrm{CW}$ heating laser for a high power pump laser (Innoslab IS8II-E, Edgewave $\mathrm{GmbH}, 20 \mu \mathrm{J}$ pulse energy), and triggered a probe laser (Innoslab BX2II-E, Edgewave GmbH, $14 \mu \mathrm{J}$ pulse energy) to generate photoacoustic signals about $300 \mathrm{~ns}$ after triggering the heating pulsed laser.

A

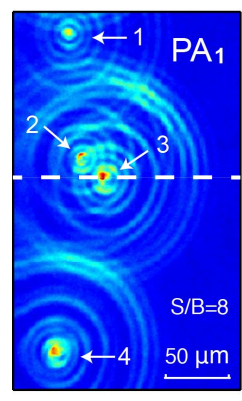

B

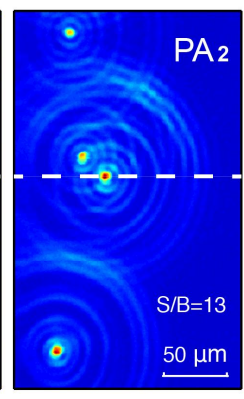

C

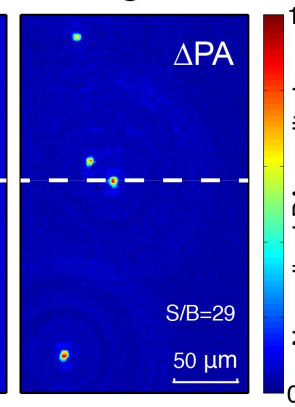

D

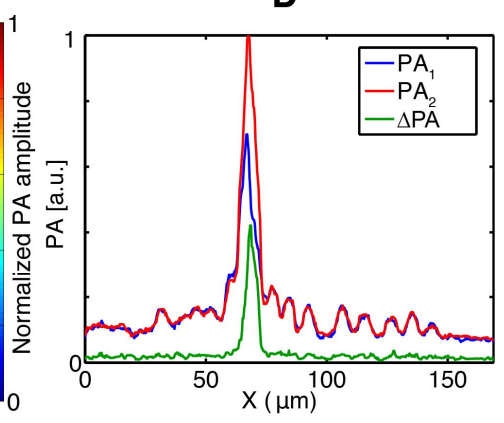

Fig. 4. Single-layer red blood cell (RBC) imaging. (A) 2D photoacoustic image $\left(\mathrm{PA}_{1}\right)$ of four RBCs in agar medium. No heating laser was applied. (B) 2D thermally enhanced photoacoustic image $\left(\mathrm{PA}_{2}\right)$ of the same $\mathrm{RBCs}$. The probe laser, triggered at $300 \mathrm{~ns}$ after the release of pulsed heating laser shot, detected the photoacoustic signal. (C). 2D differential photoacoustic image of the same RBCs. $\triangle \mathrm{PA}=\mathrm{PA}_{2}-\mathrm{PA}_{1}$. All images are normalized to the maximum amplitude of all pixels. The PA amplitude from white dashed lines in (A), (B) and (C) is compared in (D). There was no averaging for each image. (S/B: signal-to-background ratio) 
We performed two-dimensional raster scanning to image the single-layer RBC sample (actually four separated $\mathrm{RBCs}$ ) in the agar medium. The regular probe-only photoacoustic image $\mathrm{PA}_{1}$, thermally enhanced photoacoustic image $\mathrm{PA}_{2}$ and the differential photoacoustic image $\triangle \mathrm{PA}\left(\mathrm{PA}_{2}-\mathrm{PA}_{1}\right)$ are displayed in Fig. $4 \mathrm{~A}-\mathrm{C}$. We took the maximum amplitude projection (MAP) along the depth (or axial) direction to produce these images. In Fig. 4A, the regular photoacoustic image $\mathrm{PA}_{1}$ with probe-only detection rendered severely ring-shaped artifacts, which reveal the side-lobe problem in regular (linear) Bessel-beam PAM. Especially for RBC 2 and 3 as labeled in Fig. 4A, the sidelobe signals significantly interfere with other main-lobe signals. After irradiation by the heating laser, the photoacoustic signal $\mathrm{PA}_{2}$ and thereby the signal-to-background ratio were enhanced owing to the Grueneisen relaxation effect, as indicated in Fig 4B, but the side-lobe signals still exist. The maximum amplitude of $\mathrm{PA}_{2}$ was $43 \%$ greater than that of $\mathrm{PA}_{1}$ at the same position, which showed that there was a temperature rise of $13{ }^{\circ} \mathrm{C}$ in the $\mathrm{RBCs}$ due to Grueneisen effect. According to this temperature rise, the effective optical fluence around the central lobe is estimated to be $195 \mathrm{~mJ} / \mathrm{cm}^{2}$ [27]. In Fig. 4C, the differential image $\Delta \mathrm{PA}$ shows significantly reduced side-lobe signals and reveals each RBC clearly with a higher signal-to-background ratio.

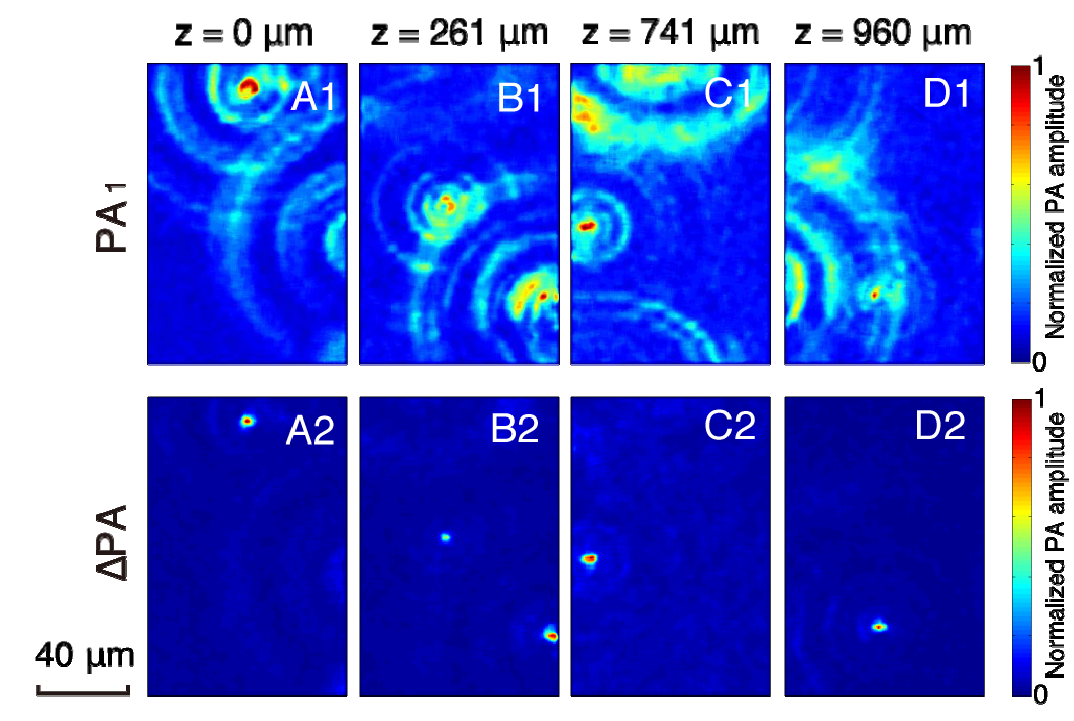

Fig. 5. 2D image slices from a volumetric image of a 1-mm-thick RBC sample in agar medium. Upper row: Regular Bessel-beam photoacoustic images $\left(\mathrm{PA}_{1}\right)$. Lower row: Nonlinear Bessel-beam photoacoustic images $(\triangle \mathrm{PA})$. Images at relative axial depths $(\mathrm{A} 1$ and $\mathrm{A} 2) \mathrm{z}=0 \mu \mathrm{m},(\mathrm{B} 1$ and $\mathrm{B} 2) \mathrm{z}=261 \mu \mathrm{m},(\mathrm{C} 1$ and $\mathrm{C} 2) \mathrm{z}=741 \mu \mathrm{m}$, and $(\mathrm{D} 1$ and $\mathrm{D} 2) \mathrm{z}=960 \mu \mathrm{m}$. The maximum signal amplitude was normalized to be 1 in each image.

Further, the nonlinear Bessel-beam PAM was used to image a thick RBC sample in agar medium. The second sample was $1 \mathrm{~mm}$ thick. Fig. 5 shows the linear $\left(\mathrm{PA}_{1}\right)$ and nonlinear $(\triangle \mathrm{PA})$ Bessel-beam photoacoustic images obtained at different depths from $\mathrm{z}=0 \mu \mathrm{m}$ to $960 \mu \mathrm{m}$. Four sets of 2D images were extracted from volumetric images of the sample. Comparing the linear $\left(\mathrm{PA}_{1}\right)$ and nonlinear $(\triangle \mathrm{PA})$ images, it is clear that the nonlinear approach was able to highlight central-lobe signals and suppress the side-lobe background over a $1 \mathrm{~mm}$ depth. For example, the image in Fig. 5-D1 shows high background arising from side lobes, almost 'burying' the actual signal from the main lobe; while the nonlinear image in Fig. 5-D2 clearly shows a clear main lobe signal. Deeper images were noisy and have weak nonlinear effects due to the limited effective focal depth of the Bessel beam in the current experimental setup. Compared with two-photon Bessel-beam microscopy [10, 25], nonlinear Bessel-beam PAM not only provides a similar long imaging depth, but also provides axial resolution along the z-axis, which is determined by the bandwidth of the ultrasonic signal. In this system, the axial resolution is estimated as $30 \mu \mathrm{m}$.

\section{CONCLUSIONS}

In summary, we have demonstrated the extended imaging depth and optical lateral resolution of nonlinear Besselbeam PAM in imaging carbon fibers and red blood cell samples. The imaging depth is determined by the effective depth of field of the Bessel beam, and the resolution is mainly determined by the central lobe diameter of the Bessel 
beam. In linear Bessel-beam PAM, the image quality is poor due to the side-lobe effect, while in nonlinear Besselbeam PAM, the side-lobe signals are effectively suppressed, thereby highlighting the main-lobe signal and offering optical lateral resolution. The experimental system can be converted to reflective mode for in vivo imaging by following the design for previous OR-PAM systems [1]. Although the optical fluence used in this work (195 $\left.\mathrm{mJ} / \mathrm{cm}^{2}\right)$ is higher than the ANSI standard $\left(20 \mathrm{~mJ} / \mathrm{cm}^{2}\right)$ but lower than the damage threshold, improving the ultrasound transducer's sensitivity to increase the signal-to-noise ratio can reduce the optical fluence. Nonlinear Bessel-beam PAM effectively extends the focal depth of photoacoustic imaging beyond that of conventional Gaussian-beam PAM, and therefore reduces the time to image thick samples.

\section{ACKNOWLEDGEMENTS}

The authors appreciate Prof. James Ballard's help with editing the manuscript. The authors also thank Konstantin Maslov, Lei Li, Jinyang Liang, and Mohammadreza Nasiriavanaki for discussions and technical help in the experiments. This work was supported in part by National Institutes of Health grants DP1 EB016986 (NIH Director's Pioneer Award), R01 CA186567 (NIH Director's Transformative Research Award), and R01 CA159959. L.V.W. has a financial interest in Microphotoacoustics, Inc. and Endra, Inc., which, however, did not support this work.

\section{REFERENCES}

[1] K. Maslov, H. F. Zhang, S. Hu, and L. V. Wang, "Optical-resolution photoacoustic microscopy for in vivo imaging of single capillaries," Optics letters, 2008.

[2] L. V. Wang and S. Hu, "Photoacoustic tomography: in vivo imaging from organelles to organs.," Science, vol. 335 , no. 6075 , pp. $1458-1462$, Mar. 2012.

[3] L. Wang, K. Maslov, and L. V. Wang, "Single-cell label-free photoacoustic flowoxigraphy in vivo.," Proceedings of the National Academy of Sciences, vol. 110, no. 15, pp. 5759-5764, Apr. 2013.

[4] L. V. Wang and L. Gao, "Photoacoustic Microscopy and Computed Tomography: From Bench to Bedside," Annu. Rev. Biomed. Eng., vol. 16, no. 1, pp. 155-185, Jul. 2014.

[5] J. Yao, L. Wang, J.-M. Yang, K. I. Maslov, T. T. W. Wong, L. Li, C.-H. Huang, J. Zou, and L. V. Wang, "Highspeed label-free functional photoacoustic microscopy of mouse brain in action," Nat Meth, vol. 12, no. 5, pp. 407-410, Mar. 2015.

[6] J. Durnin and J. J. Miceli, "Diffraction-free beams," Physical Review Letters, vol. 58, no. 15, pp. 1499-1501, Apr. 1987.

[7] J. Durnin, J. J. Miceli Jr, and J. H. Eberly, “Comparison of Bessel and Gaussian beams," Optics letters, vol. 13, no. 2 , pp. $79-80,1988$.

[8] M. R. Lapointe, "Review of non-diffracting Bessel beam experiments," Optics \& Laser Technology, vol. 24, no. 6, pp. 315-321, Dec. 1992.

[9] C. Kim, S. Park, J. Kim, S. Lee, C. Lee, M. Jeon, J. Kim, and K. Oh, "Objective-free optical-resolution photoacoustic microscopy.," J Biomed Opt, vol. 18, no. 1, pp. 10501-10501, Jan. 2013.

[10] E. J. Botcherby, R. Juškaitis, and T. Wilson, "Scanning two photon fluorescence microscopy with extended depth of field," Optics Communications, vol. 268, no. 2, pp. 253-260, Jan. 2006.

[11] T. A. Planchon, L. Gao, D. E. Milkie, M. W. Davidson, J. A. Galbraith, C. G. Galbraith, and E. Betzig, "Rapid three-dimensional isotropic imaging of living cells using Bessel beam plane illumination," Nat Meth, vol. 8, no. 5, pp. 417-423, Mar. 2011.

[12] L. Gao, L. Shao, C. D. Higgins, J. S. Poulton, and M. Peifer, "Noninvasive Imaging beyond the Diffraction Limit of 3D Dynamics in Thickly Fluorescent Specimens," Cell, 2012.

[13] G. Thériault, Y. De Koninck, and N. McCarthy, "Extended depth of field microscopy for rapid volumetric twophoton imaging," Optics Express, vol. 21, no. 8, pp. 10095-10104, Apr. 2013.

[14] G. Thériault, M. Cottet, A. Castonguay, N. McCarthy, and Y. De Koninck, "Extended two-photon microscopy in live samples with Bessel beams: steadier focus, faster volume scans, and simpler stereoscopic imaging.," Front Cell Neurosci, vol. 8, p. 139, 2014.

[15] G. Langer, K.-D. Bouchal, H. Grün, P. Burgholzer, and T. Berer, "Two-photon absorption-induced photoacoustic imaging of Rhodamine B dyed polyethylene spheres using a femtosecond laser," Optics Express, vol. 21, no. 19, pp. 22410-22422, Sep. 2013. 
[16] Y. Yamaoka, M. Nambu, and T. Takamatsu, "Fine depth resolution of two-photon absorption-induced photoacoustic microscopy using low-frequency bandpass filtering," Optics Express, vol. 19, no. 14, pp. 13365-13377, Jul. 2011.

[17] R. L. Shelton and B. E. Applegate, "Ultrahigh resolution photoacoustic microscopy via transient absorption," Biomed. Opt. Express, vol. 1, no. 2, pp. 676-686, Sep. 2010.

[18] S. P. Mattison and B. E. Applegate, "Simplified method for ultra high-resolution photoacoustic microscopy via transient absorption," Optics letters, vol. 39, no. 15, pp. 4474-4477, 2014.

[19] R. L. Shelton, S. P. Mattison, and B. E. Applegate, "Volumetric imaging of erythrocytes using label free multiphoton photoacoustic microscopy," J. Biophoton., vol. 7, no. 10, pp. 834-840, Oct. 2014.

[20] L. Wang, C. Zhang, and L. V. Wang, "Grueneisen relaxation photoacoustic microscopy.," Physical Review Letters, vol. 113, no. 17, pp. 174301-174301, Oct. 2014.

[21] A. Danielli and K. Maslov, "Label-free photoacoustic nanoscopy,” Journal of Biomedical Optics, 2014.

[22] G. Indebetouw, "Nondiffracting optical fields: some remarks on their analysis and synthesis," J. Opt. Soc. Am. A, vol. 6, no. 1, pp. 150-152, Jan. 1989.

[23] G. Scott, "Efficient generation of nearly diffraction-free beams using an axicon," Opt. Eng., vol. 31, no. 12, pp. 2640-2643, 1992.

[24] O. Brzobohatý, T. Cižmár, and P. Zemánek, "High quality quasi-Bessel beam generated by round-tip axicon," Optics Express, vol. 16, no. 17, pp. 12688-12700, 2008.

[25] G. Thériault, Y. De Koninck, and N. McCarthy, "Extended depth of field microscopy for rapid volumetric twophoton imaging.," Optics Express, vol. 21, no. 8, pp. 10095-10104, Apr. 2013.

[26] L. V. Wang and H. Wu, "Biomedical Optics: Principles and Imaging", 1st ed. WILEY-INTERSCIENCE, 2007.

[27] P. Lai, L. Wang, J. W. Tay, and L. V. Wang, "Photoacoustically guided wavefront shaping for enhanced optical focusing in scattering media.," Nature Photonics, vol. 9, no. 2, pp. 126-132, Feb. 2015. 